\title{
Editorial
}

\section{Time-Delay Systems and Their Applications in Engineering 2014}

\author{
Ligang Wu, ${ }^{1}$ Hak-Keung Lam, ${ }^{2}$ Yuxin $\mathrm{Zhao}^{3}$ and Zhan Shu ${ }^{4}$ \\ ${ }^{1}$ Space Control and Inertial Technology Research Center, Harbin Institute of Technology, Harbin 150001, China \\ ${ }^{2}$ Department of Informatics, King's College London, London WC2R 2LC, UK \\ ${ }^{3}$ College of Automation, Harbin Engineering University, Harbin 150001, China \\ ${ }^{4}$ Faculty of Engineering and the Environment, University of Southampton, Southampton SO17 1BJ, UK
}

Correspondence should be addressed to Ligang Wu; ligangwu@hit.edu.cn

Received 28 December 2014; Accepted 28 December 2014

Copyright (C) 2015 Ligang Wu et al. This is an open access article distributed under the Creative Commons Attribution License, which permits unrestricted use, distribution, and reproduction in any medium, provided the original work is properly cited.

It is well known that, together with the increasing expectations of system dynamic performances, the delay information in practical systems or processes should not be neglected in the modelling procedure. Time delay is a very common phenomenon in the engineering world. In fact, many industrial processes include after effect phenomena in their inner dynamics. And actuators, sensors, and field networks that are involved in feedback loops usually introduce unavoidable delays. The theoretical research about delay related systems should be very important, and it is surely a rewarding topic for researchers. In the past few decades, there has been an increasing interest in time-delayed systems from control scientists and engineers. On one hand, time delays are inherent in various engineering systems and often deteriorate the main system performances. Those delays can cause instabilities of original systems and make it harder for the system analysis, controller design or state estimation. To tackle these disadvantages, special attention must be paid and necessary compensation should be applied in the design process. On the other hand, the nice properties of time delays may also bring a new vision for our practical design. For example, if dealt with properly, the delay elements can really be treated as the oscillation generator or chatting compensator.

In this special issue, most papers are concerned with the stability analysis and control problems of time-delay systems. Among them, five papers investigate the simplified or improved stability criteria of time-delayed systems; seven papers consider the extended stability analysis problems of the complex systems with time delay. Based on the existing control theory, three papers discuss the output-feedback control strategies for the stochastic high-order nonlinear systems and complex interconnected time-delay systems; one paper concerns the asymptotic tracking control for a class of nonlinear systems with unknown failures of hysteretic actuators; two papers cover the optimal control problem of the nonlinear systems with time delay and the calculus variations with time delays; four papers focus on the robust $\mathscr{H}_{\infty}$ or sliding model control schemes of time-delayed dynamic systems; one paper proposes a novel fractional-order PID controller for integrated pressurized water reactor; and the adaptive and impulsive control concepts are also involved in two papers. What is more, there are five papers concerning the observer, filter, monitor, and estimation problems of timedelayed dynamic systems or processes. One paper studies the identification problem of convex function on Riemannian manifold.

Stability is the most important problem for time-delay systems. It is the basic criterion for most of time-delayed control systems. This problem has been intensively studied during the past few years. "Modified Schur-Cohn Criterion for Stability of Delayed Systems" by J. I. Mulero-Martnez gives a modified Schur-Cohn criterion for time-delay linear time-invariant systems. "New Results on Stability and Stabilization of Markovian Jump Systems with Time Delay" by H. Xia et al. deals with the problem of stochastic stability and stabilization for a class of linear Markovian jumping linear systems with discrete time-varying delay. "Improved Results on Robust Stability for Systems with Interval TimeVarying Delays and Nonlinear Perturbations" by X. Zhou et al. investigates delay-dependent robust stability criteria for systems with interval time-varying delays and nonlinear perturbations. "A Delay Decomposition Approach to the 
Stability Analysis of Singular Systems with Interval TimeVarying Delay" by J. Jiao and R. Zhang investigates delaydependent stability problem for singular systems with interval time-varying delay. "Stability Analysis and $\mathscr{H}_{\infty}$ Output Tracking Control for Linear Systems with Time-Varying Delays" by K. H. Kim et al. studies the problem of stability analysis and $\mathscr{H}_{\infty}$ output tracking control for linear systems with time-varying delays. "Fixed Points and Stability of a Class of Integrodifferential Equations" by D. Pi studies a class of integrodifferential functional differential equations with variable delay. "Robust Stability Analysis of Nonlinear Fractional-Order Time-Variant Systems" by C. Chen and Y. Xie presents a stability theorem for a class of nonlinear fractional-order time-variant systems with fractional-order $\alpha(0 \leq \alpha<1)$ by using the Gronwall-Bellman lemma. "Stability and Hopf Bifurcation of a Predator-Prey Model with Distributed Delays and Competition Term" by L.-Z. Zheng considers a class of predator-prey systems with distributed delays and competition term. "On Less Conservative Stability Criteria for Neural Networks with Time-Varying Delays Utilizing Wirtinger-Based Integral Inequality" by $\mathrm{O}$. M. Kwon et al. investigates the problem of stability analysis for neural networks with time-varying delays. "Simplified Stability Criteria for Delayed Neutral Systems" by X. Zhang et al. simplifies several existing asymptotic stability criteria in the form of linear matrix inequalities. "Stability Analysis of Fractional-Order Nonlinear Systems with Delay" by Y. Wang and T. Li studies the stability analysis of fractionalorder nonlinear systems with delay. "A Less Conservative Stability Criterion for Delayed Stochastic Genetic Regulatory Networks" by T. Yu et al. concerns the problem of stability analysis for delayed stochastic genetic regulatory networks.

In order to obtain the desired system performance, some feasible controllers must be applied to regulate the systems with time delays. "Robust $\mathscr{H}_{\infty}$ Control of Neutral System with Time-Delay for Dynamic Positioning Ships" by D. Zhao et al. proposes the less conservative result for the neutral system with state-derivative feedback by the delay decomposition approach and linear matrix inequality. "DiscreteTime Sliding Mode Control for Uncertain Networked System Subject to Time Delay" by S. C. Garcia et al. deals with uncertain systems with networked sliding mode control, subject to time delay. "Adaptive Output Feedback Sliding Mode Control for Complex Interconnected Time-Delay Systems" by $\mathrm{H}$. V. Van et al. extends the decentralized output-feedback sliding mode control scheme to stabilize a class of complex interconnected time-delay systems. "Asymptotic Tracking Control for a Class of Nonlinear Systems with Unknown Failures of Hysteretic Actuators" by C. Hou et al. proposes an adaptive failure compensation controller for a class of nonlinear systems preceded by hysteretic actuators. "A Novel Fractional-Order PID Controller for Integrated Pressurized Water Reactor Based on Wavelet Kernel Neural Network Algorithm" by Y. Zhao et al. presents a novel wavelet kernel neural network with wavelet kernel function. "Quasipolynomial Approach to Simultaneous Robust Control of TimeDelay Systems" by N. Semenič et al. investigates a control law for retarded time-delay systems, concerning infinite closed-loop spectrum assignment. "Research on Adaptive
Neural Network Control System Based on Nonlinear UModel with Time-Varying Delay" by F. Xu et al. proposes a new approach, namely, U-model approach, to solve the problems of analysis and synthesis for nonlinear systems. "Optimal Control with Time Delays via the Penalty Method" by M. Benharrat and D. F. M. Torres proves necessary optimality conditions of Euler-Lagrange type for a problem of the calculus of variations with time delays, where the delay in the unknown function is different from the delay in its derivative. "Robust Moving Horizon $\mathscr{H}_{\infty}$ Control of Discrete Time-Delayed Systems with Interval Time-Varying Delays" by F. Y. Tascikaraoglu et al. considers the design of a delaydependent type moving horizon state-feedback control for a class of linear discrete-time systems subject to time-varying state delays, norm bounded uncertainties, and disturbances with bounded energies. "Time-Delayed Impulsive Control of Chaotic System Based on T-S Fuzzy Model" by C. Hu and H. Jiang is concerned with the time-delayed impulsive control and synchronization of general chaotic system based on T-S fuzzy model. "Optimal Control of a Nonlinear Time-Delay System in Batch Fermentation Process" by Y. Yu considers an optimal control problem involving a nonlinear time-delay system to get a high yield of products in batch process. "Output-Feedback Control for a Class of Stochastic HighOrder Feedforward Nonlinear Systems with Delay" by Q. Ma et al. investigates the problem of global output-feedback stabilization for a class of stochastic high-order time-delay feedforward nonlinear systems with different power orders. "Adaptive Fuzzy Output-Feedback Method Applied to Fin Control for Time-Delay Ship Roll Stabilization" by R. Bai considers the ship roll stabilization by fin control system and investigates the adaptive fuzzy output-feedback control.

Some specific problems about observer, estimation, monitoring, filtering, and identification of time-delay systems and industrial processes are very important. These topics have been analyzed separately. "Observer Design for One-Sided Lipschitz Nonlinear Systems Subject to Measurement Delays" by S. Ahmad et al. presents a novel nonlinear observerdesign approach to one-sided Lipschitz nonlinear systems in the presence of output delays. "Estimation of Nonlinear Functions of State Vector for Linear Systems with TimeDelays and Uncertainties" by I. Y. Song et al. focuses on estimation of a nonlinear function of state vector in discretetime linear systems with time delays and model uncertainties. " $\mathscr{H}_{\infty}$ Filtering for Discrete Markov Jump Singular Systems with Mode-Dependent Time Delay Based on T-S Fuzzy Model" by C. Gong and Y. Zeng investigates the $\mathscr{H}_{\infty}$ filtering problem of discrete singular Markov jump systems with mode-dependent time delay based on T-S fuzzy model. "Monitoring of Nonlinear Time-Delay Processes Based on Adaptive Method and Moving Window" by Y. Fan et al. proposes a new adaptive KPCA algorithm for monitoring nonlinear time-delay process. "Experimental Evaluation of FSM-Based Testing Cost for Time-Delay Systems" by Z. Wei et al. presents a timer embedded FSM model to design a comparative strategy for assessing the coverage criteria and test suites generation methods for time-delay systems. " $\mathscr{H}_{\infty}$ Filtering for Networked Systems with Bounded Measurement Missing" by $\mathrm{H}$. Yang et al. is concerned with the $\mathscr{H}_{\infty}$ filtering 
problem for networked systems with bounded measurement missing. "The Identification of Convex Function on Riemannian Manifold" by L. Zou et al. presents the identification of convex function on Riemannian manifold using the Penot generalized directional derivative and the Clarke generalized gradient.

Many practical applications related with time delay are also concerned with. "Approximate Design of Optimal Disturbance Rejection for Discrete-Time Systems with Multiple Delayed Inputs: Application to a Jacket-Type Offshore Structure" by S.-Y. Han et al. is concerned with the problem of optimal disturbance rejection for a class of discretetime systems with multiple delayed inputs. "The Impact of Awareness Programs with Recruitment and Delay on the Spread of an Epidemic" by L. Zuo et al. discusses the impact of awareness programs on the spread and control of infectious diseases in a given region. "The Application of Time-Delay-Dependent $\mathscr{H}_{\infty}$ Control in the Transmission Effect of Monetary Policy on Real Estate Market" by H. Guo et al. uses a time-delay-dependent $\mathscr{H}_{\infty}$ control method to analyze the transmission effect of monetary policy on the real estate market. "Modelling and Simulation of Volume Controlled Mechanical Ventilation System" by Y. Shi et al. derives a new mathematical model of the ventilation system. "Robust Predictive Control of Water Level in an Experimental Pilot Plant with Uncertain Input Delay” by F. Q. Rossi and R. K. H. Galvão is concerned with the predictive control of water level in an experimental pilot plant, in the presence of input constraints and uncertain time delays. "Study of Robust $\mathscr{H}_{\infty}$ Filtering Application in Loosely Coupled INS/GPS System" by L. Zhao et al. gives an illustration of minimum mean square Kalman filter in integration GPS/INS system and a contrast with traditional Kalman filter. "Modeling and Bifurcation Research of a Worm Propagation Dynamical System with Time Delay" by Y. Yao et al. adopts both vaccination and quarantine strategy to control the internet worm propagation. "Global Existence and Uniqueness of Solutions for a Free Boundary Problem Modeling the Growth of Tumors with a Necrotic Core and a Time Delay in Process of Proliferation" by $\mathrm{S}$. Xu and M. Huang studies a mathematical model for the growth of necrotic tumors with time delays in proliferation.

For both the theoretical and practical impact, time-delay systems have been an enduring theme in the study of differential equations, stochastic processes, statistics theory, and systems theory. These research topics have broad applications to a number of areas such as mechanical, electrical, and chemical engineering. And the theoretical research results about time-delayed systems have played an important role in solving these delay related problems. Recently, some open problems about time delay have also emerged, such as using the delayed inputs to stabilize and regulate the original system, adaptive identification of delays in practical problems, taking advantage of the stochastic properties of time delay, and considering the delay information for observers. Needless to say, these open problems are both challenging and rewarding for theoretical researchers and practical engineers. And many articles in this special issue have been concerned with these most recent theoretical developments and practical applications.
Of course, the selected topics and papers are not a comprehensive representation of the area of this special issue. Nonetheless, they represent the rich and many-faceted knowledge and we hope the readers will find them well readable and useful.

\section{Acknowledgments}

We would like to express our great appreciation to the authors for their remarkable contributions in assisting us. Finally, all reviewers' fundamental work on these papers is also very greatly acknowledged.

Ligang $W u$

Hak-Keung Lam Yuxin Zhao

Zhan Shu 


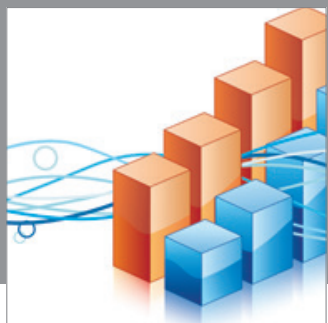

Advances in

Operations Research

mansans

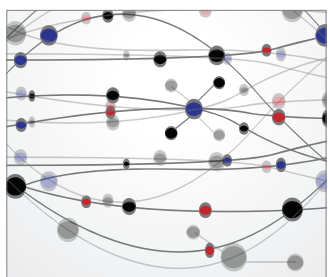

The Scientific World Journal
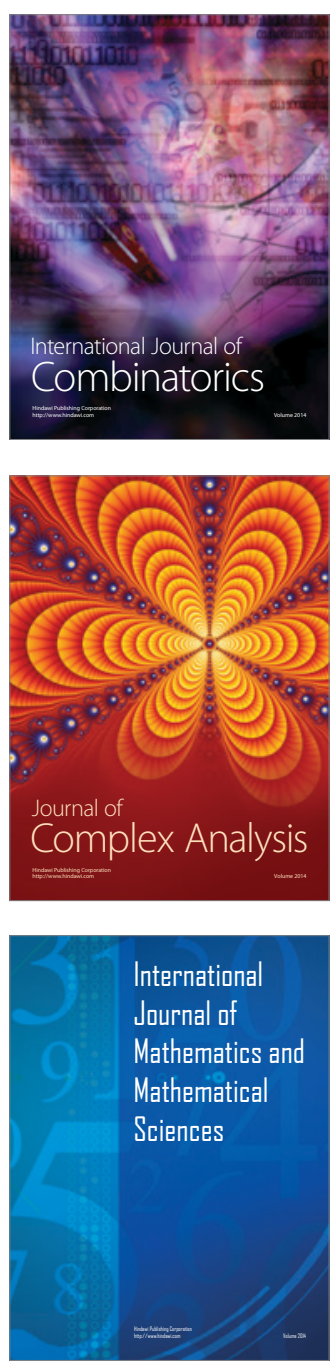
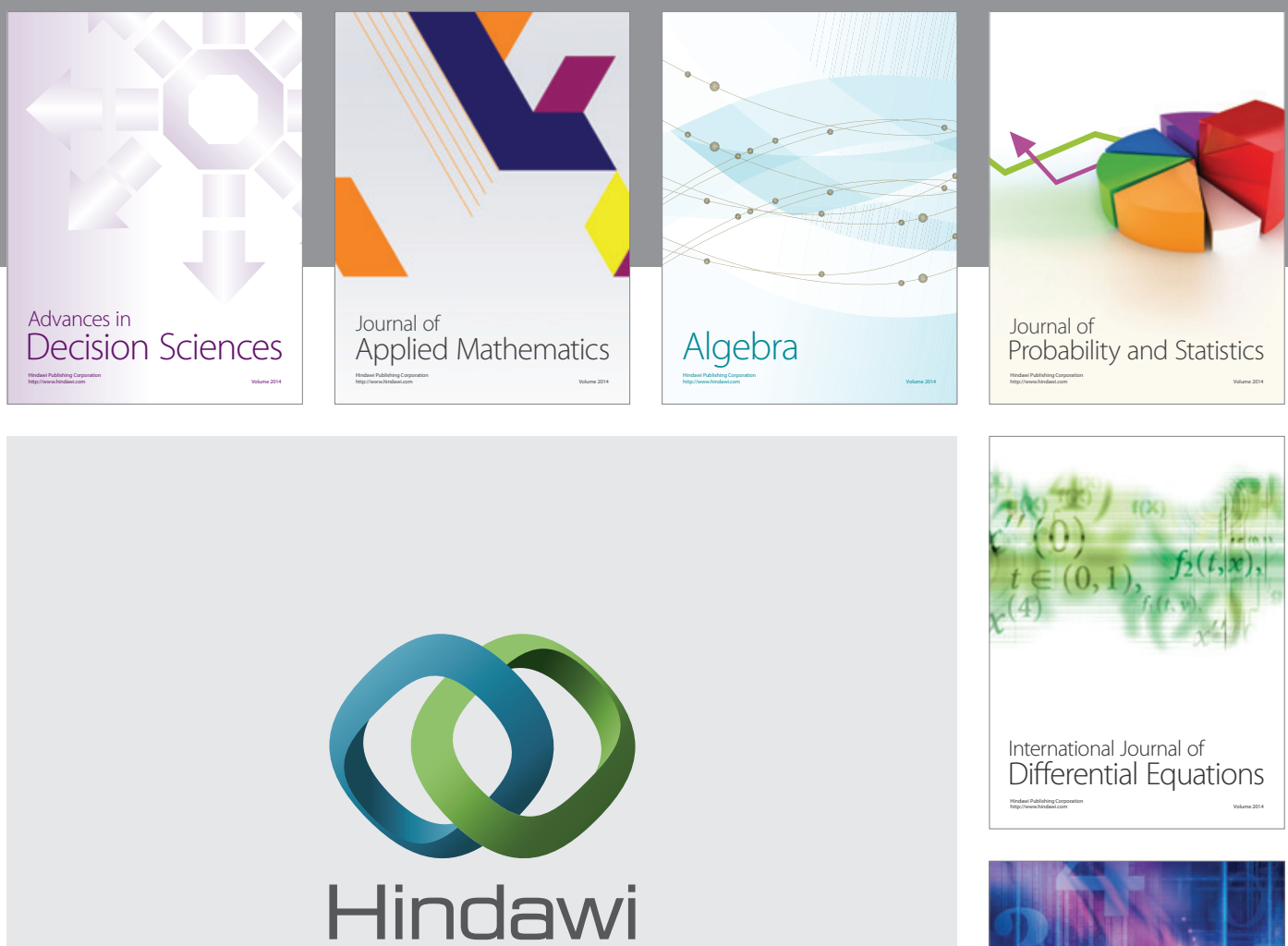

Submit your manuscripts at http://www.hindawi.com
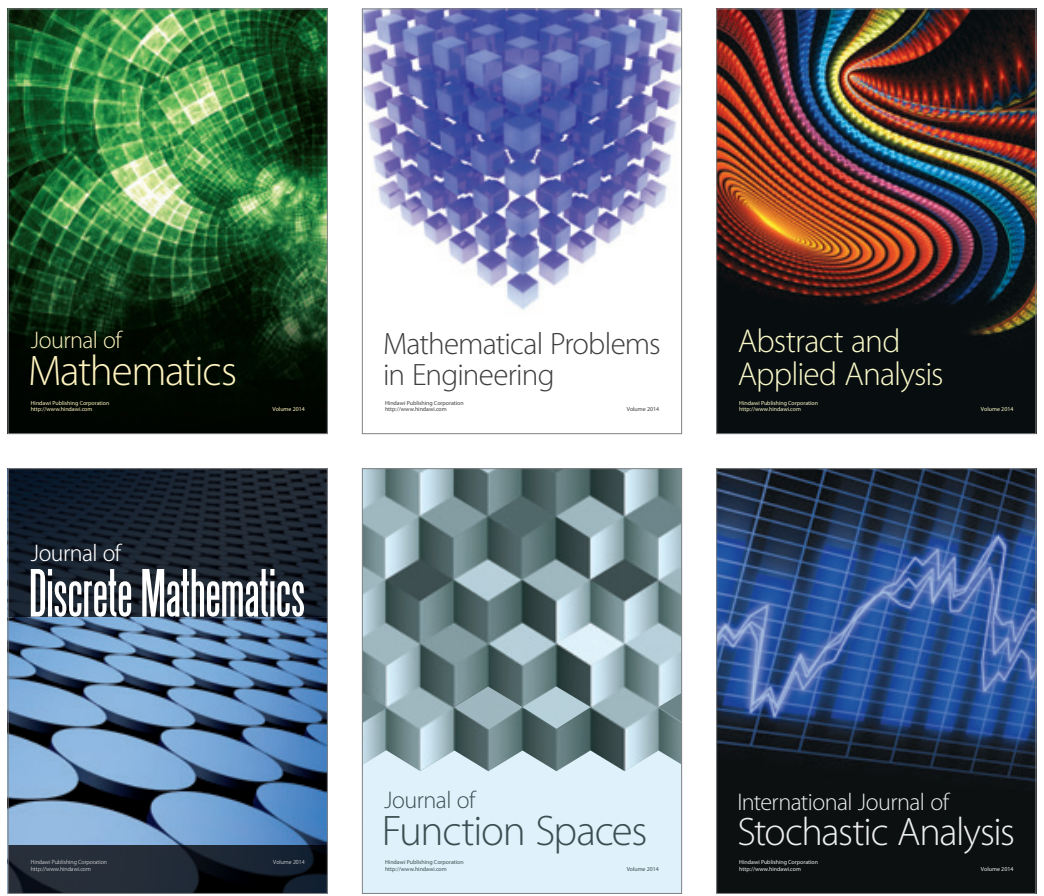

Journal of

Function Spaces

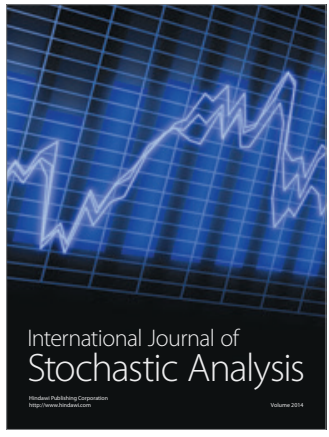

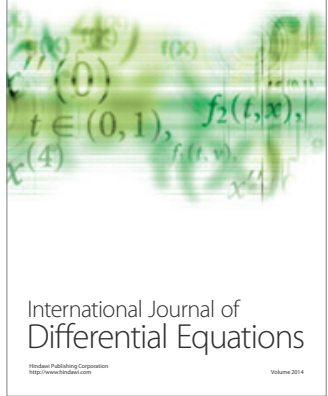
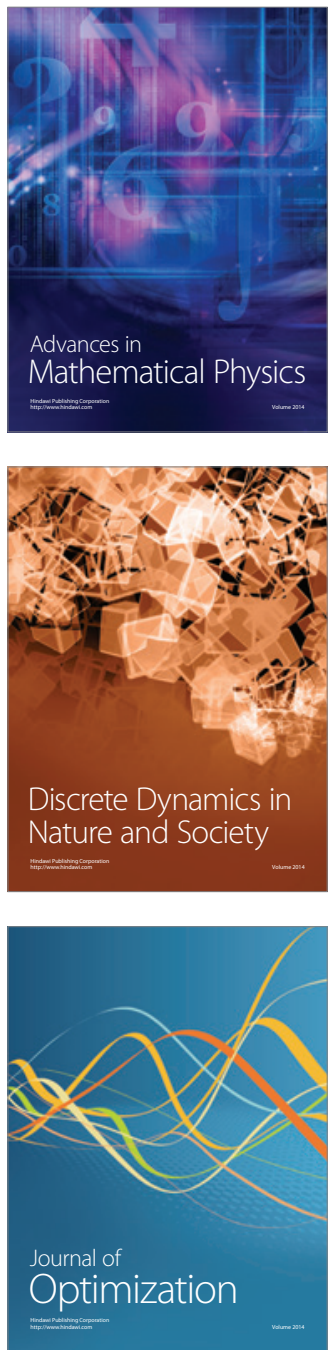